\title{
A 2.4-GHz CMOS Down-Conversion Doubly Balanced Mixer with Low Supply Voltage
}

\author{
Chih-Chun Tang, Wen-Shih Lu, Lan-Da Van and Wu-Shiung Feng \\ Lab. 328, Department of Electrical Engineering, National Taiwan University \\ Taipei, Taiwan, ROC \\ E-mail: tang1216@seed.net.tw
}

\begin{abstract}
A CMOS down-conversion mixer with the combination of Gilbert Cell mixer and modified low voltage design technique using LCtank is demonstrated in this paper. The RF, LO and IF port frequencies are $2.4 \mathrm{GHz}, 2.3 \mathrm{GHz}$ and $100 \mathrm{MHz}$, respectively. The measurement results of the proposed mixer exhibit $6.7 \mathrm{~dB}$ of conversion gain, $-18 \mathrm{dBm}$ of $\mathrm{P}_{-1 \mathrm{~dB}}$ compression point and $-7.5 \mathrm{dBm}$ of IIP3 with $-8 \mathrm{dBm}$ LO power and $1.8 \mathrm{~V}$ supply voltage. The power consumption in mixer core is $5.94 \mathrm{~mW}$. This mixer was fabricated in 0.35 um 1P4M CMOS process and the size is $1.5 \mathrm{X}$ $1.1 \mathrm{~mm}^{2}$. It can provide $0.7 \mathrm{~dB}$ conversion gain even though $1.3 \mathrm{~V}$ supply voltage is utilized.
\end{abstract}

\section{INTRODUCTION}

In the past few years, with the growing popularity of mobile PCS. GPS, and wireless-LAN, the wireless communication business has become a big market. The manufacturers have to integrate system in order to reduce the size, weight. and cost of the products. Many researches have explored the characteristics of CMOS in radio frequency (RF) [1.2] such that deep sub-micron CMOS has became an attractive candidate in the applications of low-GHz frequency range. Unfortunately, many wireless products have short stand-by time or too heavy for hand-held due to their large batteries. Hence, low power consumption is the key design issue of wireless portable products.

Fig. 1 shows the typical front-end building blocks of a superhetrodyne receiver. Because the characteristics of image rejection filter significantly depend on output loading. the input impendence of RF port should match well to the output impendence of the image rejection filter. In order to reduce the noise figure (NF) of the overall system, the mixer should have low NF and adequate conversion gain to minimize the noise contribution of the IF stage. On the other hand. the conversion gain should not be too large because a strong signal may saturate the output of the mixer and reduce its power gain. The input $1 \mathrm{~dB}$

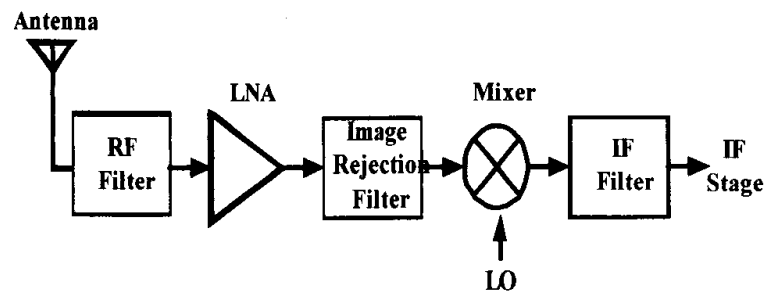

Figure 1 The RF front-end of the superhetrodyne receiver.

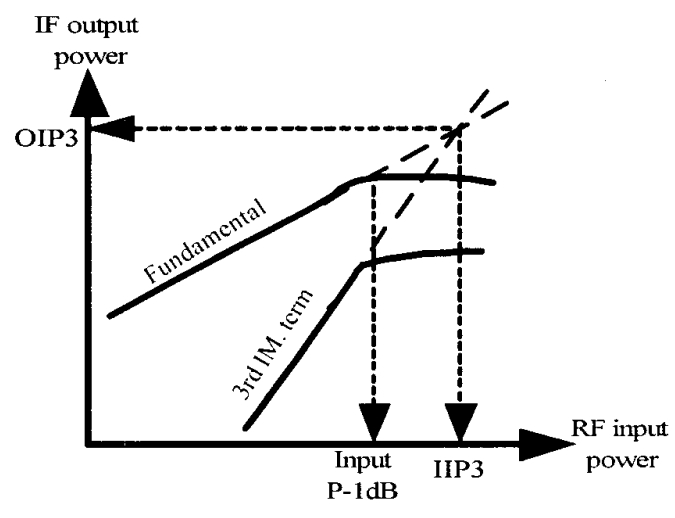

Figure 2 The definitions of input P-1dB. IIP3 and OIP3.

compression point $\left(\mathbf{P}_{-1 \mathrm{~dB}}\right)$ measures the input power level that causes the output power deviate from its linear magnitude response by $1 \mathrm{~dB}$. Also the thirc-order inter-modulation (IM3) product generated by two undesired adjacent channel signals characterizes another parameter of mixer linearity, called third-order intercept point (IP3). The definitions of the input $\mathrm{P}_{\mathrm{ldB}}$. input third-order intercept point (IP3) and output third-order intercept point (OIP3) are illustrated in Fig. 2. The mixer is desired to have higher linearity. so higher input $\mathrm{P}-1 \mathrm{~dB}$ and IIP3 are preferred.

In terms of conversion gain, mixers can be divided into two types. which are passive and active. The advantage of the passive mixers is better linearity, but the disadvantages are conversion loss. higher $\mathrm{NF}$ and larger LO power. In contrast to the passive mixers. the active mixers provide conversion gain. lower NF and demarid smaller LO power. A smaller LO power requirement is very important in mixer design. There are several reasons. First. it is difficult to generate large LO power in low voltage and low power design. Second. larger LO power means larger LO-to-RF feedthrough, which results in LO signal leaks through the antenna and becomes a strong interference to other RF systems. Third. reducing the required $L O$ power also indicates that improve the LO-RF and LO-IF isolation.

\section{CMOS Mixer Design}

\subsection{Gilbert Cell Mixer}

Fig. 3 is the conventional doubly balanced CMOS Gilbert Cell mixer [3]. The transconductance stage consists of $\mathrm{M} 1$ and $\mathrm{M} 2$, ard current commutating stage consists of M3. M4. M5 and M6. 


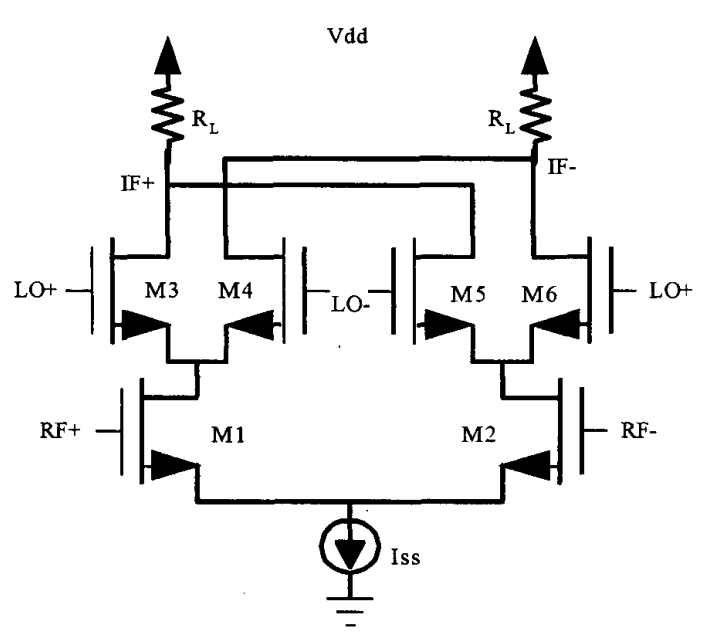

Figure 3 The simplified CMOS Gilbert Cell mixer.

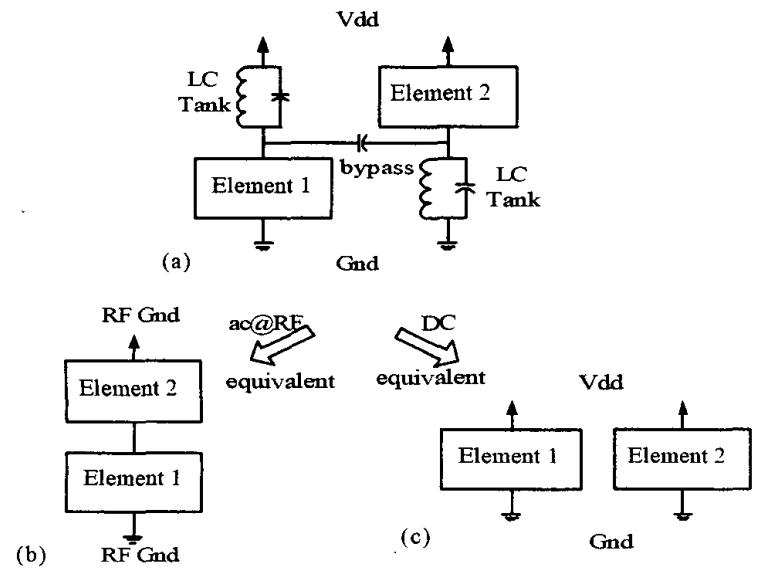

Figure 4 The low voltage topology using LC tanks and bypass capacitor (a) the complete topology, (b) RF equivalent circuit. and (c) DC equivalent current.

$R_{L}$ is the load resistor. The voltage gain (Av), which is given by Eq. 1 , is determined by the transconductance of $\mathrm{M} 1$.

$$
A v=g_{m} R_{L} \frac{2}{\pi}
$$

Since all transistors operate in saturation region. the expected drain to source voltage is $V_{T}+200 \mathrm{mV}$. neglecting body effect. For a typical $\mathrm{V}_{\mathrm{T}}$ of $0.7 \mathrm{~V}$, this traditional Gilbert Cell mixer needs at least $2.7 \mathrm{~V}$ supply voltage to operate.

\subsection{Low Voltage Architecture with LC Tanks}

A low voltage design technique for RF IC was proposed in [4]. and Fig. 4 shows this concept. It consists of two LC tanks and one bypass capacitor. The two LC tanks resonate at the desired RF frequency to provide an infinite impendence ideally.

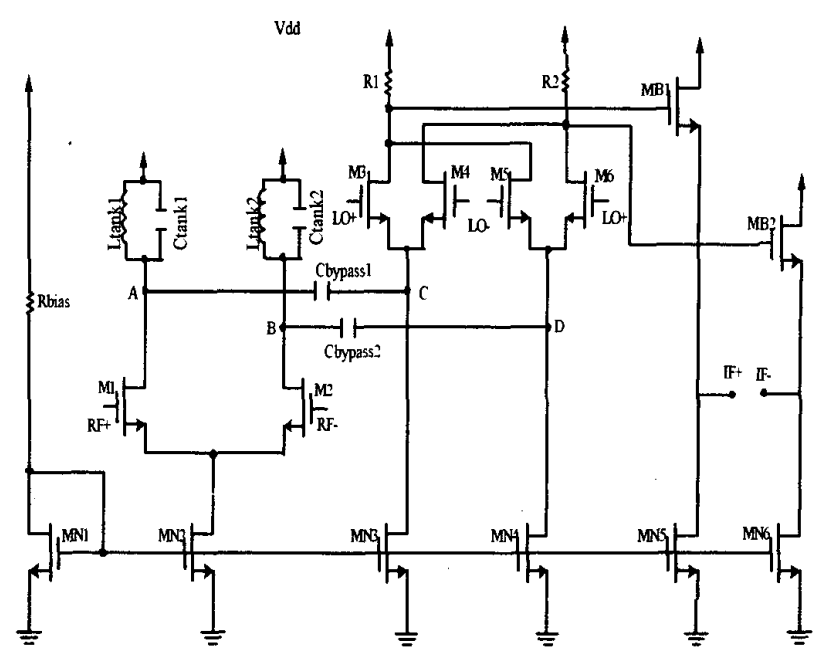

Figure 5 The simplified schematic of the proposed mixer.

The bypass capacitor is responsible for coupling the RF signal between two branches. At DC, the $\mathrm{LC}$ tank requires no voltage headroom. and there is only one element between supply voltage and ground in each branch.

\subsection{Proposed CMOS Mixer}

The proposed CMOS mixer is shown in Fig. 5. The quality factor (Q) of on-chip spiral inductor is very low $(<5)$ due to the CMOS lossy substrate. Assume Rs is the parasitic resistor of the inductor. the equivalent resistance of the LC tank at resonance, which denoted as $\mathrm{Rr}$, is given by Eq. 2 .

$$
R r=R s\left(1+Q^{2}\right)
$$

ASITIC [5] is used to design and simulate the on-chip inductors. If $\mathrm{L}$ is chosen to be $4 \mathrm{nH}$, the quality factor is about 4 and the parasitic resistor is $10 \mathrm{Ohm}$ in this $0.35 \mathrm{um} \mathrm{CMOS}$ process. From Eq. 2. the $\mathrm{Rr}$ is calculated to be about $170 \mathrm{Ohm}$. This result shows the equivalent impendence of $\mathrm{LC}$ tank at parallel resonance is not infinite due to the low quality factor of the on-chip inductor. If we directly apply the LC tanks to Gilbert Cell mixer, there should four LC tanks. However, the output resistance of MN3 and MN4 are aboutt 2.5KOhm in this circuit and much larger than $170 \mathrm{Ohm}$. So. $\mathrm{MN} 3$ and MN4 are used to replace the two LC tanks, which one is connected from node $\mathrm{C}$ to ground and the other is connected from node $\mathrm{D}$ to ground. Thus the mixer still works properly with smaller chip area. The MB1 and MB2 are added as buffers for measurement to drive $50 \mathrm{Ohm}$.

As compared the Gilbert Cell mixer with the proposed mixer, the first advantage of the proposed mixer is the reduction of the stacked transistors. That means it is more suitable for low voltage application than traditional Gilbert Cell mixer.

As shown in Fig. 3. the larger bias current is needed to improve the conversion gain. The larger bias current causes the larger voltage drop on R1 and R2, and thus decrease the voltage headroom of the 
remaining stacked MOSFETs and degrade the linearity of the mixer. Another advantage of the poposed mixer is to allow the designer to easily adjust the bias current in the transconductance stage without affecting the current-commutating stage. This is a particular important improvement in the mixer design.

The transconductances of $\mathrm{M} 1$ and $\mathrm{M} 2$ are set to $13 \mathrm{mS}$. The overdrive voltages, $\left(\mathrm{Vgs}-\mathrm{V}_{\mathrm{T}}\right)$, of $\mathrm{M} 3$ to $\mathrm{M} 6$ are all about $70 \mathrm{mV}$ to reduce the required LO power and make sure M3 to M6 switch between saturation and cutoff region.

\section{Simulation and Measurement Results}

\subsection{MOSFET Model and Simulation}

There are many parasitic effects in RF design and the origina model provided by foundry is not accuracy enough in such high frequency. The modified MOSFET model [6] is used to simulate the circuit. The bias range of this model is $0 \sim 3 \mathrm{~V}$ of $V_{D S}$ and $0 \sim 2 \mathrm{~V}$ of $\mathrm{V}_{\mathrm{GS}}$. The frequency range is from $50 \mathrm{MHz}$ to $20.05 \mathrm{GHz}$.

Sweep the LO power from $-30 \mathrm{dBm}$ to $+3 \mathrm{dBm}$ to find out the conversion gain and the required LO power. The RF input signal power is set to be $-47 \mathrm{dBm}$ at $2.4 \mathrm{GHz}$. The conversion gain is about $7.6 \mathrm{~dB}$ when LO power is $-8 \mathrm{dBm}$. To ensure the mixer still provides the conversion gain when process deviation occurs. the other process comers are also used to simulate the same circuit. The result is shown in Fig. 6.

\subsection{Measurement Setup and Results}

The die is mounted on FR4 board for testing. The first step is to measure the S-parameters of the RF ports for designing the matching networks. After matching is completed. the test signal is differentially fed into RF and LO ports. The 180-degree lumped element hybrid is designed to convert the single-ended signal to differential signal. The measured unbalance amplitude is $0.18 \mathrm{~dB}$ at $2.3 \mathrm{GHz}$ and $0.16 \mathrm{~dB}$ at $2.4 \mathrm{GHz}$. The phase difference is 180.3 degree at $2.3 \mathrm{GHz}$ and 180.5 -degree at $2.4 \mathrm{GHz}$. The testing board is shown in Fig. 7.

We set the RF power to $-50 \mathrm{dBm}$ and LO power to $-8 \mathrm{dBm}$ on signal generators. Fig. 8 is the IF output frequency spectrum of the single tone testing. The RF signal loss of cables and 180-degree hybrid is measured together to be $2.4 \mathrm{~dB}$, and the loss of cable on IF port is $0.5 \mathrm{~dB}$. So. the measured conversion gain is $6.7 \mathrm{~dB}$. After sweeping the LO power from $-30 \mathrm{dBm}$ to $+8 \mathrm{dBm}$. the conversion gain is measured and also shown in Fig. 6.

The reason of the difference in conversion gain between simulation and measurement results is the LO port does not match to $50 \mathrm{ohm}$ perfectly while LO power is lower than $-10 \mathrm{dBm}$.

LO power is set to $-8 \mathrm{dBm}$ and perform sweep of RF power from $-57.5 \mathrm{dBm}$ to $-12.5 \mathrm{dBm}$ to measure the linearity of the proposed mixer. As shown in Fig. 9. the simulation result of input $P$. $1 \mathrm{~dB}$ is $-19.5 \mathrm{~dB}$ and the measurement result is $-18 \mathrm{~dB}$. Fig. 10 shows the two tones testing result of the proposed mixer.

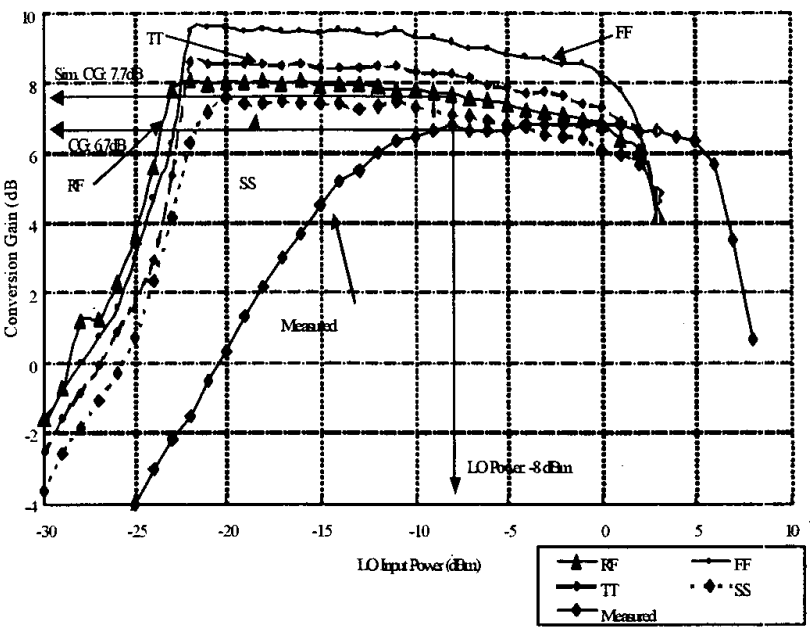

Figure 6 The simulation and measurement results of conversion gain v.s LO power of the proposed mixer.

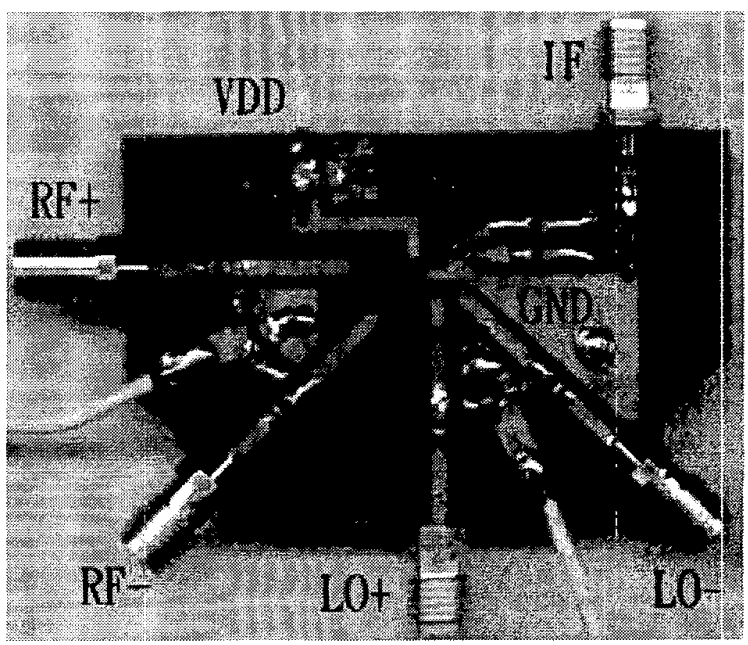

Figure 7 The test board of the proposed mixer.

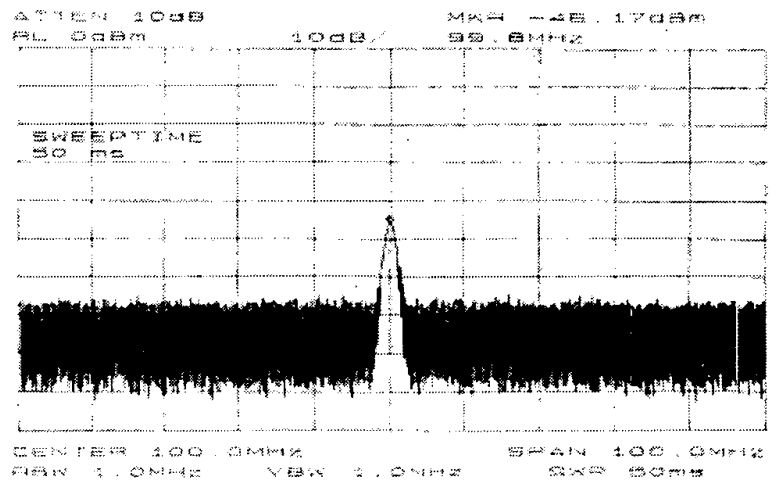

Figure 8 The IF port output frequency spectrum of single tone testing. 
The RF input tones are $2400 \mathrm{MHz}$ and $2401 \mathrm{MHz}$ and $\mathrm{LO}$ is $2300 \mathrm{MHz}$. Thus the fundamental terms of the IF output are $100 \mathrm{MHz}$ and $101 \mathrm{MHz}$. The third-order inter-modulation terms are $99 \mathrm{MHz}$ and $102 \mathrm{MHz}$. Fig. 11 shows the simulated and measured IIP3 results of the proposed mixer. The measured linearity is better than the simulation result because the measured conversion gain is slightly lower than the simulation result.

\section{SUMMARY}

The characteristics of the proposed mixer are summarized in Table 1. According to the measurement results, the proposed mixer provides $6.7 \mathrm{~dB}$ conversion gain under the $1.8 \mathrm{~V}$ supply voltage and it also demonstrates $0.7 \mathrm{~dB}$ conversion gain for supply woltage as low as $1.3 \mathrm{~V}$. The potential for low voltage applications of the proposed mixer is proved. The proposed mixer can be easily integrated with other front-end circuits to build CMOS transceiver.

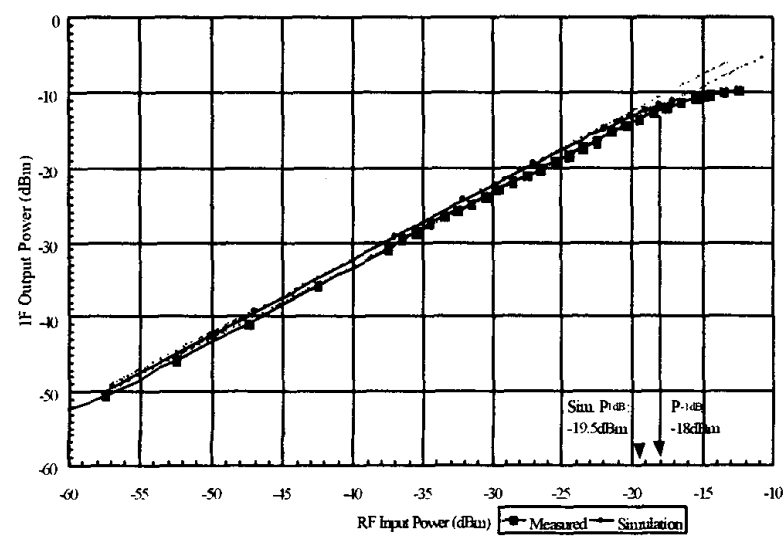

Figure 9 The simulated and measured results of $P_{-1 d B}$ of the proposed mixer.

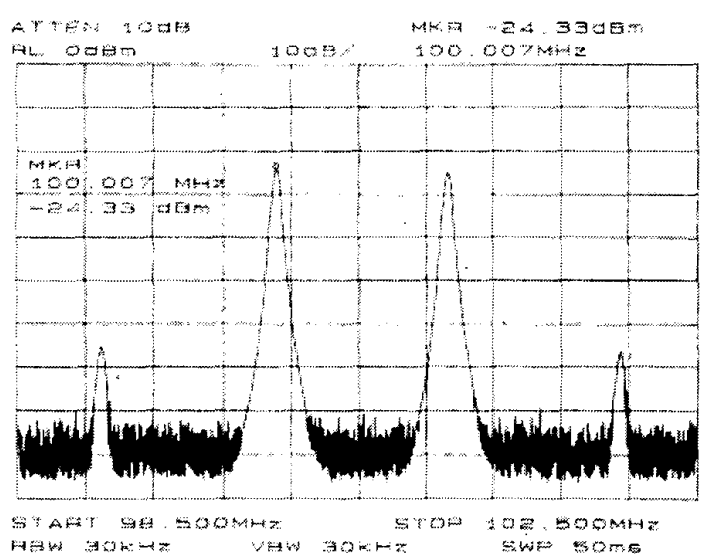

Figure 10 The IF port output frequency spectrum of two tones testing.

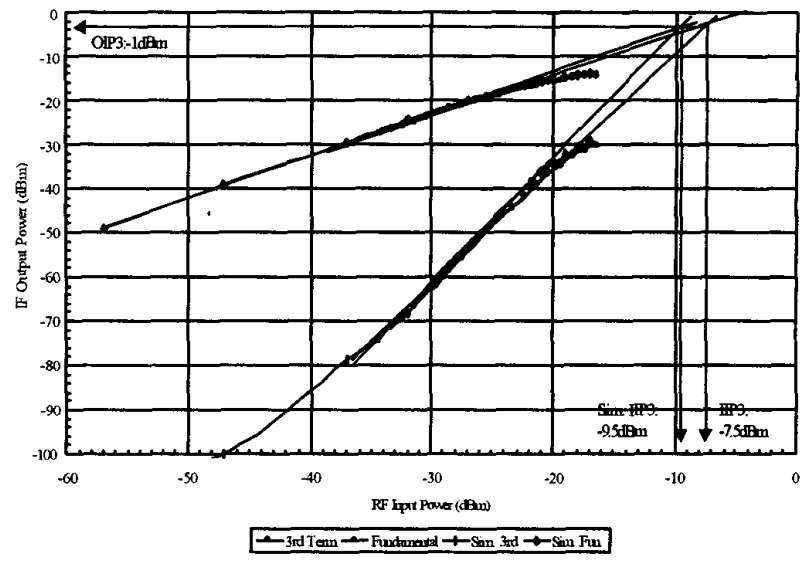

Figure 11 The simulated and measured results of IIP3 and OIP3.

Table 1 The proposed mixer performance.

\begin{tabular}{|c|c|}
\hline RF $=$ 2.4GHz and LO $=\mathbf{2 . 3 G H z}$ & Measured Results \\
\hline LO power (dBm) & -8 \\
\hline Conversion gain (dB) & 6.7 \\
\hline IIP3 (dBm) & -7.5 \\
\hline P $_{\text {-1dB }}(\mathbf{d B m})$ & -18 \\
\hline Current in mixer core (mA) & 3.3 \\
\hline Supply voltage (V) & 1.8 \\
\hline Power consumption (mW) & 5.94 \\
\hline Port-to-port isolation (dB) & $>20$ \\
\hline Chip size (mm X mm) & $1.1 \times 1.5$ \\
\hline Process & TSMC 0.35um 1P4M \\
\hline
\end{tabular}

\section{REFERENCES}

[1] Q: Huang. F. Piazza, P.Orsatti, and T. Ohguro "The Impact of Scaling Down to Deep Submicron on CMOS RF Circuits" IEEE Journal of Solid-State Circuits. vol. 33, no.7, pp.10231036, July 1998.

[2] T. Manku "Microwave CMOS-Device Physics and Design" IEEE Journal of Solid-State Circuits, vol. 34, no.3, pp277. 285. March 1999.

[3] B. Gilbert "A precise four quadrant multiplier with subnanosecond response" IEEE Journal of Solid-State Circuits. vol. SC-3, pp.365-373. Dec. 1968.

[4] T. Manku, G. Beck, and E. J. Shin "A Low-Voltage Design Technique for RF Integrated Circuits" IEEE Trans. Circuits Syst.--Part II , vol. 45, no.10. pp1408-1413. Oct. 1998.

[5] A. M. Niknejad and R. G. Meyer "Analysis, Design, and Optimization of Spiral Inductors and Transformers for Si RF IC's" IEEE Journal of Solid-State Circuits. vol. 33. no.10. pp1470-1481. Oct. 1998.

[6] C. C. Hsiao, C. W. Kuo. and Y. J. Chen " $6.8 \mathrm{GHz}$ monolithic oscillator fabricated by 0.35 um CMOS technologies" Electronics Letters, vol. 36. no.23, pp. 1927-1928. Nov. 2000. 